\title{
Image Fusion using Non Subsampled Contourlet Transform in Medical Field
}

\author{
Jampani Ravi, M. Gowri Sri Durga, Y. D. R. Ch. Kartheek, MD. Shabeena Begum, T. Raju, T. V. Syamala \\ Raju
}

Abstract: Image fusion is a powerful method and developing field in the area of image processing. The image fusion is a type of methodology that combines the two or more images into single more informative image. Image fusion is the process of assimilation of numerous input images into a new single fused image with highly informative than the input image. There are various image fusion transform techniques are proposed. Out of that techniques a Non-subsampled Counterlet transform includes shift invariant property, highly directionality, reduced the cost and more efficient information as compared to previous techniques such as wavelet transform(WT), DWT, LWT, MWT, CWT, Curvelet transform, Contourlet transform. In NSCT, we decompose the images into low frequency and high frequency using sparse representation and absolute-maximum rule respectively. The DGSR algorithm is used for the better performance of SR-based approach. Finally, to reconstruct the image we use inverse NSCT and output is fused image.

Keywords : Image fusion, NSCT, SPARSE, SENSOR.

\section{INTRODUCTION}

The image fusion is the process of assembling all the information from various images and their incorporation into a single fused image. The resultant fused image gives information which is more effective and precise than given input images and it contains all the required information. The theme of image fusion technique is not only for the reduction of amount of data but also to construct images that are convenient and easy to understand for the human and machine perception. Applications where image fusion used are remote sensing, satellite imaging, medical field etc. In

Revised Manuscript Received on February 15, 2020.

* Correspondence Author

Jampani Ravi*, Assistant Professor, Department of ECE, SRKR Engineering College, Bhimavaram, India. Email: jravi472@gmail.com

M. Gowri Sri Durga, Student, Department of ECE, SRKR Engineering

College, Bhimavaram, India. Email: gowrisridurga034@gmail.com

Y. D. R. Ch. Kartheek, Student, Department of ECE, SRKR Engineering College, Bhimavaram, India.

Email: charankartheek75@gmail.com

MD. Shabeena Begum, Student, Department of ECE, SRKR

Engineering College, Bhimavaram, India.

Email: shabeenamd1321@gmail.com

T. Raju, Department of ECE, SRKR Engineering College, Bhimavaram, India, Email: rajutalluri99999@gmail.com

T. V. Syamala Raju, Assistant Professor, Department of ECE, SRKR Engineering College, Bhimavaram, India. Email: tvsyam@gmail.com

(c) The Authors. Published by Blue Eyes Intelligence Engineering and Sciences Publication (BEIESP). This is an open access article under the CC BY-NC-ND license (http://creativecommons.org/licenses/by-nc-nd/4.0/) this paper we are dealing with the Image fusion in Medical field.

In medical field, there are two types of imaging. They are Structural Imaging and Functional Imaging. The methods such as Positron Emission Tomography (PET), Single Photon Emission Computed Tomography (SPECT), Computed Tomography (CT), Functional Magnetic Resonance (MRI), Functional Photo Acoustic Microscopy (FPAM), Magnetic Particle Imaging (MPI), and Optical Imaging.

\section{DIFFERENT TYPES OF IMAGE FUSION}

The types of image fusion i.e., Single Sensor Imaging and Multi Sensor Imaging.

Single Sensor Imaging: In Single Sensor Imaging, the images are captured by only one sensor but it may not capture always provide complete information of the particular target. By using Single sensor the images may be capture on same modalities. In single sensor it takes more time to capture the image and at different distances it cannot be focused at same time.

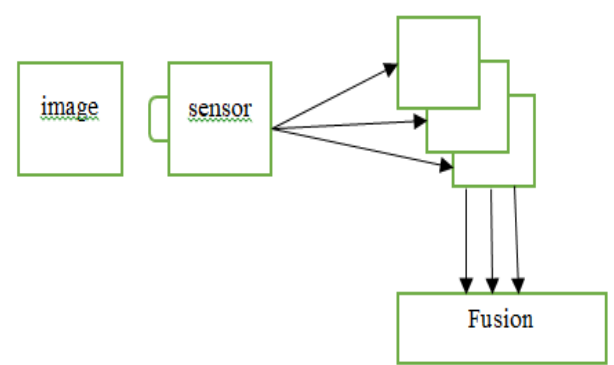

Fig 1: Single Sensor Image Fusion

Multi Sensor Imaging: In Multi Sensor Imaging, the images are captured by two or more sensors. It takes the less time to capture the two or more images of same scene at same time. By using the multi sensor the images are captured on different modalities. Multi Sensor Imaging is more effective and captures more information than the Single Sensor Imaging. 


\section{Image Fusion using Non Subsampled Contourlet Transform in Medical Field}

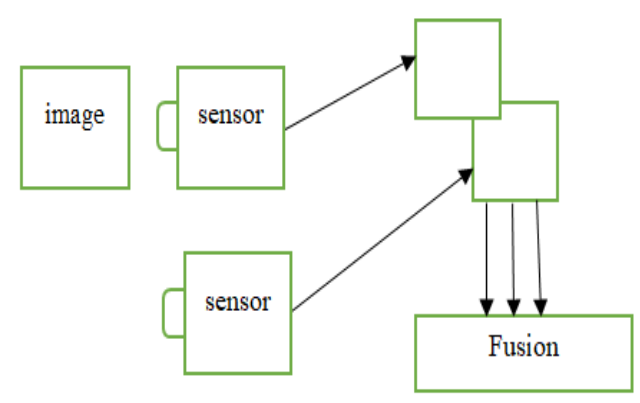

Fig 2: Multi Sensor Image Fusion

\section{IMAGE FUSION TECHNIQUES}

There are different techniques which are introduced in image fusion. The techniques are Wavelet Transform, Curvelet Transform, Contourlet Transform, and Nonsubsampled Contourlet Transform (NSCT).

In Wavelet Transform, Wavelet allows decomposition of complex information like music, speech, images and patterns into elementary forms at various positions, scales and subsequently reconstructed with high precision. There are different types in wavelet transform. They are Stationary Wavelet Transform, Discrete Wavelet Transform (DWT), Lifted Wavelet Transform (LWT), Morlet Wavelet Transform (MWT), Complex Wavelet Transform (CWT), Dual Tree Complex Wavelet Transform (DT-CWT). The major drawbacks in the wavelet transform, it does not provide shift invariance. In Discrete wavelet transform (DWT) has poor directionality and fails to represent curvilinear structures. SWT and DT-CWT cannot capture curves and edges of images. To overcome these drawbacks we go for Curvelet Transform.

In Curvelet Transform, there are two types i.e., unequally spaced fast Fourier transform and wrapping based fast curvelet transform. It can be used for both continuous and digital domain. The advantages are high directivity, curves edges efficiently, reduce noise effects and represents edges better than wavelet transform. But in the Curvelet transform, the disadvantages are shift variance and it is not associated with multi-resolution analysis. To overcome these drawbacks we go for contourlet transform.

In Contourlet Transform, the advantages are Multi-resolution, discrete domain implementation, multidirectional, reduces the redundancy. But in the Counterlet transform, the disadvantages are Shift variant, it leads to Pseudo Gibbs phenomenon, frequency selectivity and temporal stability are poor.

To overcome these drawbacks in the previous techniques we proposed the Non-subsampled Counterlet transform (NSCT). In NSCT, the advantages are Shift Invariance in decomposition process, and effective Infrared and Visible Image Fusion scheme and reduce the cost.

\section{NON SUBSAMPLED CONTOURLET TRANSFORM}

NSCT is based on counterlet transform which eliminates the sampling process during the image decomposition and reconstruction. The advantages of NSCT are Shift invariance, Multiresolution. We can obtain multidimensionality of an image using Non Sampled filter bank. While the NSCT is proposed in image fusion, we can get more information and reduces the effects of inaccurate on the fused results can also be reduced effectively. Hence the NSCT is better technique for image fusion when compared to all other techniques. The NSCT consists of two parts. They are Non Sampled Pyramid (NSP), Non Subsampled Directional Filter Banks (NDFB). The first source image is decomposed by NSP with different scales to get the sub band coefficients at different scales. Those subband coefficients are decomposed by NSDFB to obtain different scales and different directions. In NSCT multiscale property is obtained by using dual channeled Non Subsampled two dimensional filter banks for sub band decomposition to be done. The directional filter bank NSCT is constructed by merging critically sampled dual channeled fan filter banks and resampling processes. The NSDFB is used to remove downsamplers and upsamplers in the DFB to obtain shift invariance directional expansion. In NSCT decomposition the image fusion is decomposed into low pass and high pass sub bands.

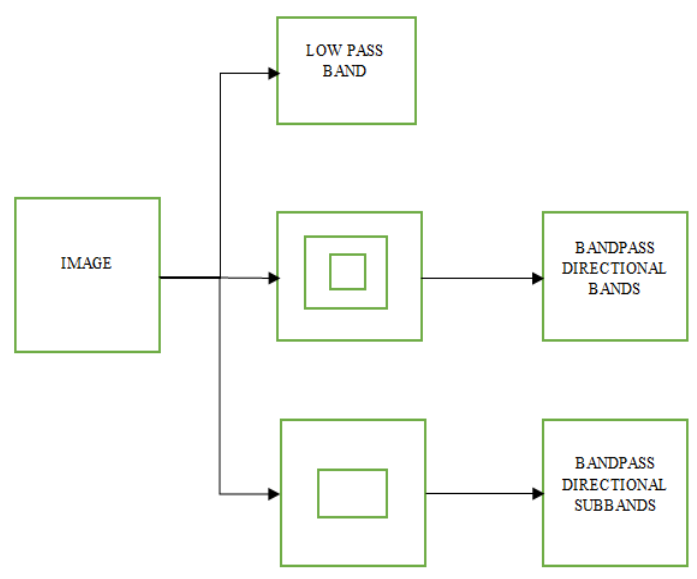

Fig 3: Non Subsampled Contourlet Transform

\section{SPARSE REPRESENTATION}

Sparse Representation, in this the main theme deals with obtaining sparse solutions for systems of linear equations. At different scales and orientations, we have a different sub band images of NSCT. By training subdictionaries separately for each band, contain all the information. We achieve it, the information at different scales and orientation.

The low pass subband coefficients have large amplitude and contain more information. The low frequency components used with sparse representation (S.R) based fusion approach. In this we take the sparse coding for stability and efficiency. To achieve this, we divide the source images into image patches for low frequency image fusion scheme. We proposed the dynamic group sparsity recovery (D.G.S.R) algorithm to improve the performance of S.R-based approach. The algorithm that purnes data residues in the iterative process according to both sparsity and group clustering trend. Therefore the D.G.S.R algorithm is better than previous algorithm is orthogonal matching pursuit (O.M.P).

\section{Published By:}

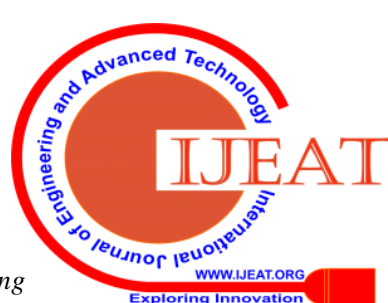


The high pass subband coefficients have small amplitude and less information. To achieve the better, we use the high frequency components by using the absolute maximum rule which are already used in counterlet transform.

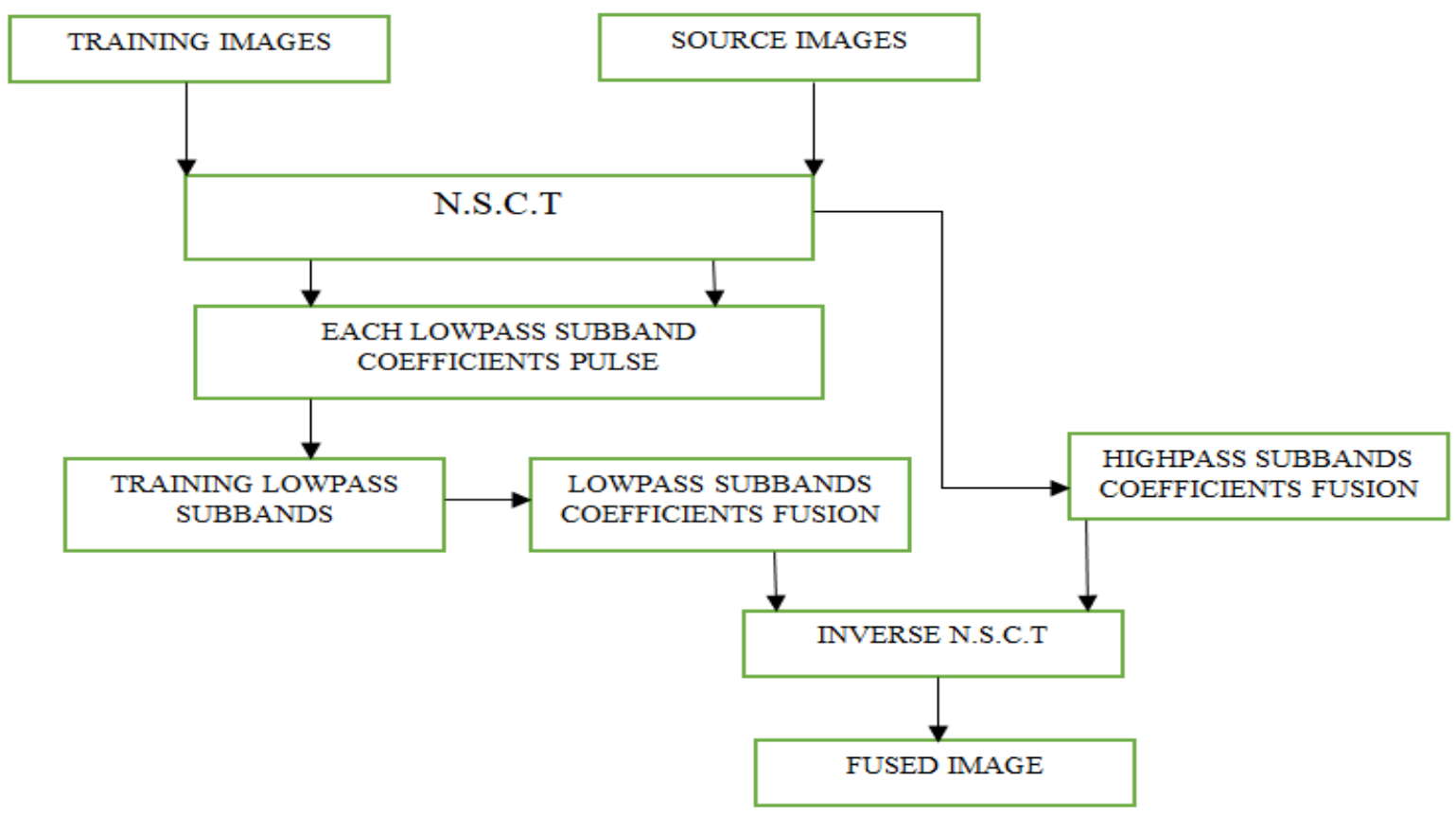

Fig 4: Flow chart of the Non Subsampled Contourlet Transform (NSCT)

VI. EXPERIMENTAL RESULTS

(a)

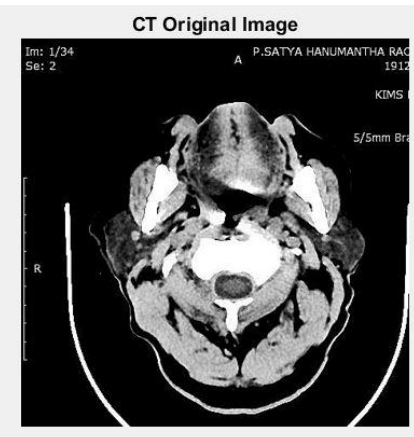

MRI Original Image

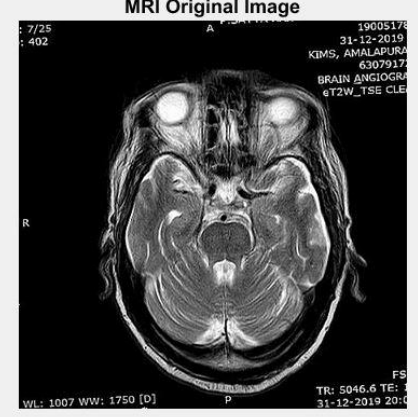

fused image

(b)

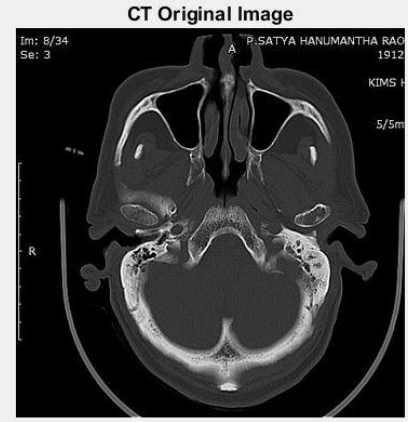

MRI Original Image
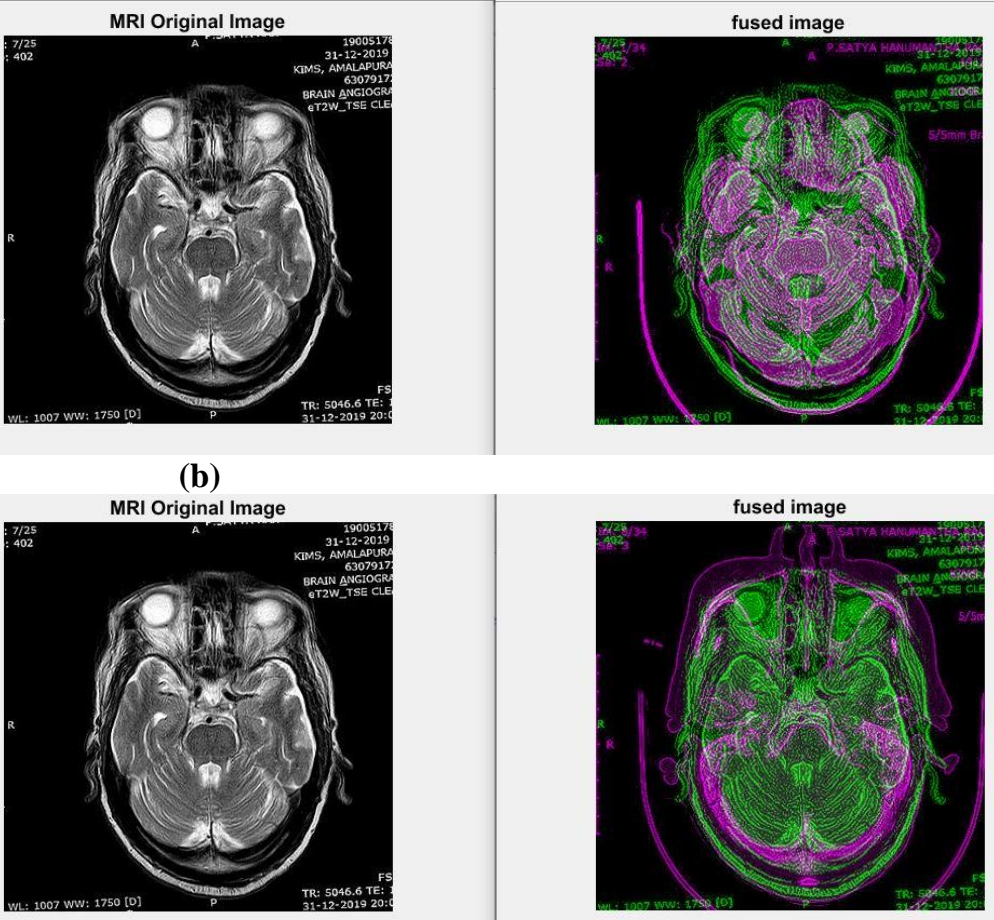

fused image
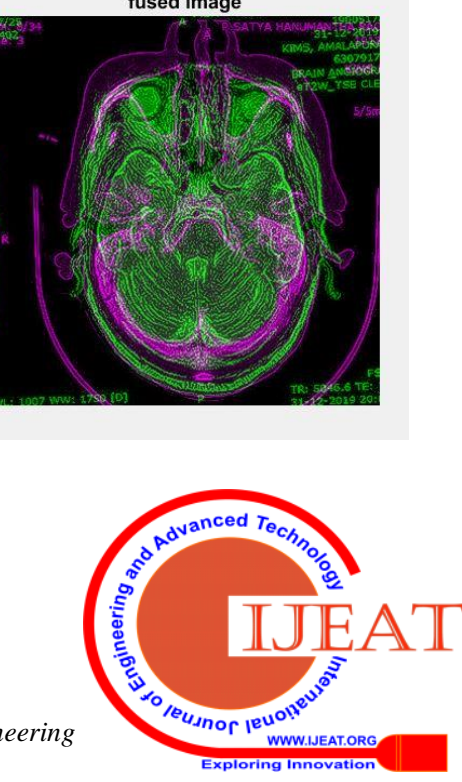
(c)
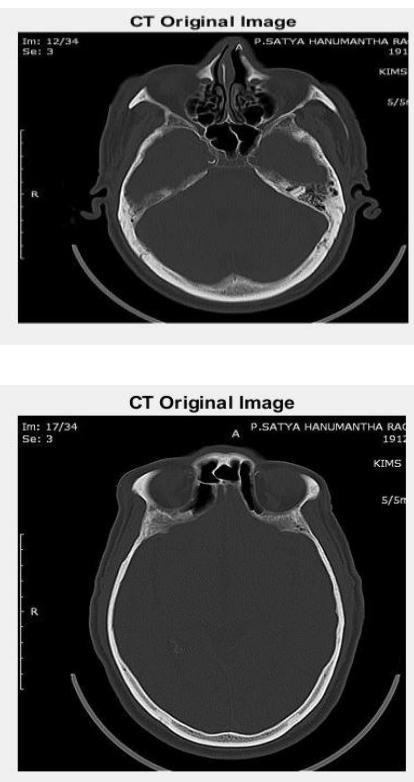

MRI Original Image

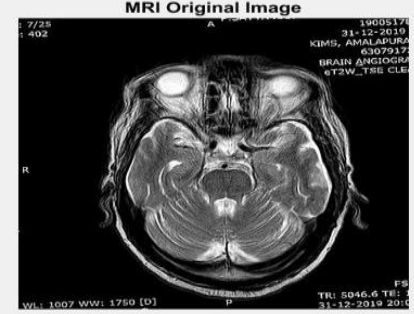

(d)

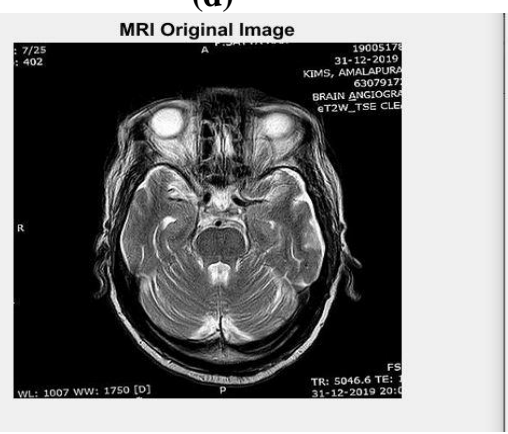

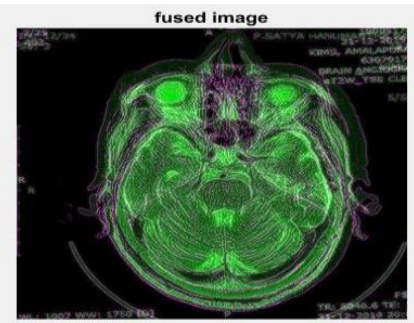

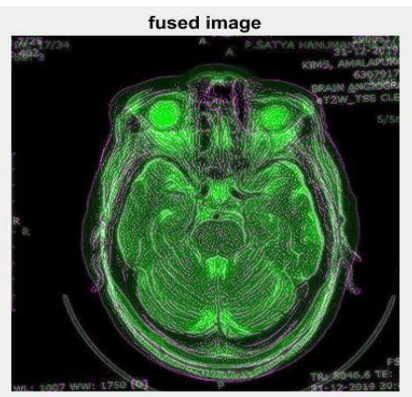

\section{CONCLUSION}

In the image fusion the Non Subsampled Contourlet Transform (NSCT) is better technique as compared to the previous technique. There are many advantages on NSCT. In NSCT, it avoids the Gibbs phenomenon which occurs in Counterlet Transform and also it has shift invariant property. In this NSCT, we use the DGSR algorithm in low frequency fusion to achieve the images be effectively and feasible. The advantages of NSCT is we eliminate the downsampling and upsampling and reduce the effects of misregistration on the fused image effectively. The other advantages of NSCT are highly directionality, reduced cost, highly feasible and the more efficient information. Finally we conclude that the image fusion The Non Subsampled Contourlet Transform (NSCT) technique is better technique when compared to the previous techniques like wavelet, CWT, LWT, DWT, MWT, Curvelet and Contourlet Transform.

\section{REFERENCES:}

1. J. Ravi, M. Praveen Kumar, "Segmentation of 3D MRI Images of the Brain using a PCA Atlas", International of Engineering Science \& research Technology 6(10..., 2017.

2. J. Ravi, K. Venkat rao, N. Kishore Chandra Dev, k. Yugandhar," Survey on Image Segmentation Using Thresholding methods", International Journal for research and Development in Technology 8 (4), 130-137, 2017.

3. J. Ravi, K. Venkat rao, N. Kishore Chandra Dev, "Implementation of Image Encryption Using Elliptic Curve Cryptography”, International Journal of Scientific Progress \& Research 39 (1), 13-16, 2017.

4. J. Ravi, K. Venkat rao, "Implementation of Image Resolution Enhancement", International of Engineering Science \& research Technology 6(9..., 2017.

5. J. Ravi, "Image Fusion Algorithm Implementation Using Laplacian Pyramid", International Journal of Scientific Progress \& Research 29 (3), 103-107, 2016.

6. J. Ravi, P. subba Rao, G. Soma Lakshmi, S. Mahesh Babu, N. Priyanka, "Image Segmentation Based on Background Recognition and Edge Detection", ICAST, 73, 2019.

7. J. Ravi, M. Gowri Sri Durga, MD. Shabeena Begum, Y.D.R.C. Kartheek, T. Raju, "Implementation of Image Fusion using Matlab", International Journal of Science \& Advance Research in Technology 5 (4...,2019.

8. J. Ravi, P. subba Rao, G. Soma Lakshmi, S. Mahesh Babu, N. Priyanka, "Image Segmentation Based on Background Recognition and Edge Detection", International Journal of Science \& Advance Research in Technology 5 (4..,2019.

9. J. Ravi, N.V. Phani sai Kumar," Image Compression Implementation using Discrete Wavelet Transform", International Journal of Science \& Advance Research in Technology 5 (11), 2023, 2018.

\section{AUTHORS PROFILE}

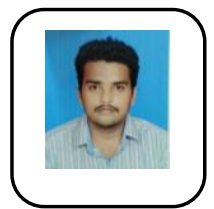

Jampani Ravi, Presently working as an Assistant Professor in ECE of S.R.K.R. Engineering college. He is presently doing his part-time Ph. D in Sathyabama Institute of Science \& Technology, Chennai in the area of Digital Image Processing. He received his M. Tech degree from Andhra University in Communication Systems. He received his B. Tech degree from JNTUA in ECE. His research interest lies in the area of Digital Image Processing, Digital Signal Processing.

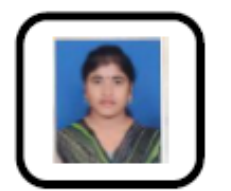

M. Gowri Sri Durga Presently studying 4/4 B. Tech in ECE SRKR Engineering college, Bhimavaram, India. Her research interest lies in the area of Microprocessors And Microcontrollers \& Digital Electronics.

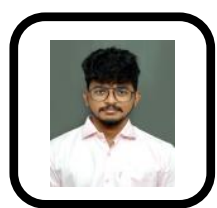

Y. D. R. CH. Kartheek, Presently studying $4 / 4$ B. Tech in ECE SRKR Engineering college, Bhimavaram , India. His research interest lies in the area of Digital Electronics \& Digital Image Processing.

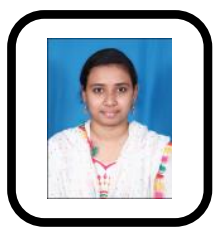

MD. Shabeena Begum, Presently studying 4/4 B. Tech in ECE SRKR Engineering college, Bhimavaram , India. Her research interest lies in the area of Digital Image Processing \& Cellular Mobile Comuunication.

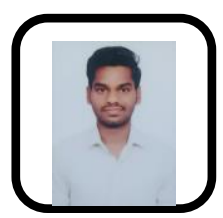

T. Raju, Presently studying 4/4 B. Tech in ECE SRKR Engineering college, Bhimavaram , India. . His research interest lies in the area of . of Switching Theory And Logical Design \& Digital Image Processing.

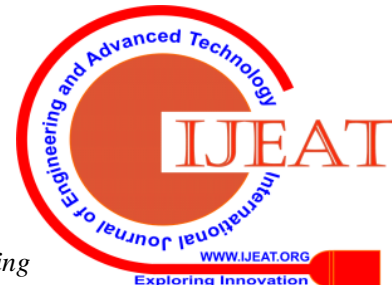

